\title{
Impact de la varicocélectomie chez les patients ayant une azoospermie non obstructive ou une oligozoospermie sévère
}

\section{Impact of varicocelectomy in patients with nonobstructive azoospermia or severe oligozoospermia}

\author{
B. Fall $\cdot$ B. Diao $\cdot$ Y. Sow $\cdot$ A. Sarr $\cdot$ P.A. Fall $\cdot$ A.K. Ndoye $\cdot$ M. Ba $\cdot$ B.A. Diagne \\ Reçu le 21 mai 2010 ; accepté le 5 juillet 2010 \\ (C) SALF et Springer-Verlag France 2010
}

Résumé But : Évaluer les résultats de la ligature haute rétropéritonéale des veines spermatiques chez les hommes infertiles ayant une azoospermie non obstructive ou une oligozoospermie sévère.

Patients et méthodes : Étude rétrospective colligeant 61 cas de varicocèle dont $46(75,4 \%)$ ayant une oligozoospermie sévère (groupe I) et $15(24,6 \%)$ ayant une azoospermie non obstructive (groupe II).

Résultats : Dans le groupe I (oligozoospermie sévère), l'âge moyen des patients était de 35,5 $\pm 6,4$ ans (23-47 ans). La durée moyenne de l'infertilité était de 4,9 $\pm 3,4$ ans (1-13 ans). La varicocèle était bilatérale chez 41 patients $(89,1 \%)$ et unilatérale gauche chez cinq patients $(10,9 \%)$. Cette varicocèle a été classée de grade I dans deux cas $(4,3 \%)$, de grade II dans 39 cas $(84,7 \%)$ et de grade III dans cinq cas $(10,9 \%)$. En préopératoire, la concentration moyenne des spermatozoïdes était de 1,85 $\pm 1,4$ millions $/ \mathrm{ml}$. La mobilité normale à la première heure des spermatozoïdes était en moyenne de 43,3 $\pm 21,5 \%$. Les spermatozoïdes de morphologie anormale représentaient $65,05 \pm 21,6 \%$ en moyenne. Après varicocélectomie, la concentration moyenne des spermatozoïdes était de $8,3 \pm 10,3$ millions $/ \mathrm{ml}$. La mobilité normale était en moyenne de 47,6 $\pm 29,2 \%$, et les spermatozoïdes de morphologie anormale de 50,08 $\pm 26,9 \%$. Après un recul moyen de 26,2 $\pm 11,6$ mois, 12 couples $(26,1 \%)$ avaient rapporté des grossesses spontanées. Dans le groupe II (azoospermie), l'âge moyen des patients était de $40,8 \pm 7,2$ ans (27-47 ans). La durée moyenne de l'infertilité était de $6 \pm 3,1$ ans (3-15 ans). Après varicocélectomie, une induction de la spermatogenèse avec présence de spermatozoïdes mobiles dans l'éjaculat a été observée chez trois patients $(20 \%)$. Dans ce groupe, un seul couple avait

B. Fall $(\bowtie) \cdot$ B. Diao $\cdot$ Y. Sow · A. Sarr · P.A. Fall · A.K. Ndoye

M. Ba B.A. Diagne

Service d'urologie-andrologie, CHU Aristide-Le-Dantec,

Dakar, Sénégal

e-mail : bbcrfall@yahoo.fr rapporté une grossesse qui s'est terminée par une fausse couche.

Conclusion : La varicocélectomie chez les hommes infertiles ayant une azoospermie non obstructive peut entraîner une apparition dans l'éjaculat de spermatozoïdes mobiles utilisables dans le cadre d'une assistance médicale à la procréation (AMP). Elle entraîne une amélioration significative de la qualité de la spermatogenèse et de la fertilité en cas d'oligozoospermie sévère. La réalisation de cette intervention chez de tels hommes est donc une option raisonnable. Pour citer cette revue : Androl. 20 (2010).

Mots clés Infertilité · Varicocélectomie · Azoospermie non obstructive $\cdot$ Oligozoospermie sévère

Abstract Objective: To evaluate the changes induced by retroperitoneal varicocelectomy on infertile men with nonobstructive azoospermia or severe oligozoospermia.

Patients and methods: The records were retrospectively evaluated for 46 infertile men with severe oligozoospermia (group I) and 15 infertile men with non-obstructive azoospermia (group II). The parameters of sperm before and after surgery and unassisted pregnancy rate were comparatively analysed.

Results: In the severe oligozoospermia group, the mean age of patients was $35.5 \pm 6.4$ (23-47 years). The mean duration of infertility was $4.9 \pm 3.4$ years (1-13 years). Of these patients, 41(89.1\%) had bilateral varicocele and five $(10.9 \%)$ had unilateral left-side varicocele. The varicocele was classified as grade I in two cases $(4.3 \%)$, grade II in 39 cases $(84.7 \%)$ and grade III in five cases (10.9\%). After surgery, the mean sperm count increased from $1.85 \pm 1.4$ to $8.3 \pm 10.3$ millions $/ \mathrm{ml}$ and mean sperm normal motility from $43.3 \pm 21.5$ to $47.6 \pm 29.2 \%$. The mean sperm abnormal morphology decreased from $65.05 \pm 21.6$ to $50.08 \pm$ $26.9 \%$. After a mean follow-up of $26.2 \pm 11.6$ months, the unassisted pregnancy rate in this group was $26.1 \%$. In the non-obstructive azoospermia group $(N=15)$, the mean age 
of patients was $40.8 \pm 7.2$ (27-47 years). The mean duration of infertility was $6 \pm 3.1$ years (3-15 years). After varicocelectomy, an induction of spermatogenesis was observed in three patients $(20 \%)$ with presence of motile sperm in the ejaculate. In this group, only one of the 15 men achieved unassisted pregnancy.

Conclusion: Retroperitoneal varicocele repair resulted in spermatogenesis induction with presence of motile ejaculated spermatozoa for some men with non-obstructive azoospermia. It induced spermatogenesis and fertility enhancement in men with severe oligozoospermia. Varicocele repair should be considered in men with non-obstructive azoospermia or severe oligozoospermia. To cite this journal: Androl. 20 (2010).

Keywords Infertility · Varicocelectomy $\cdot$ Nonobstructive azoospermia $\cdot$ Severe oligozoospermia

\section{Introduction}

L'infertilité masculine a été pendant longtemps un sujet tabou dans les sociétés d'Afrique subsaharienne où l'infertilité du couple était toujours imputée à la femme. Ces dernières années, elle est de plus en plus comprise et acceptée par ces populations. C'est pourquoi elle constitue actuellement au Sénégal un des plus fréquents motifs de consultation dans les services d'urologie et d'andrologie.

La varicocèle est la plus fréquente anomalie physique observée chez les hommes infertiles. De nos jours avec les progrès en imagerie médicale, elle est mise en évidence chez un quart à un tiers des hommes infertiles [1]. Elle est une cause majeure d'infertilité [2], et la ligature de la veine spermatique est généralement considérée comme le traitement de choix de la varicocèle [3]. Mais du fait de la rareté des études contrôlées, la relation causale entre varicocèle et infertilité masculine est insuffisamment mise en évidence [4], et les effets de la varicocélectomie chez les hommes infertiles ayant une oligozoospermie sévère ou une azoospermie non obstructive font toujours l'objet de controverses.

Dans la première étude sur l'utilité de la varicocélectomie chez l'homme infertile publiée en 1952, Tulloch [5] rapportait une grossesse spontanée obtenue après cure de varicocèle chez un homme ayant une azoospermie. Avec le développement des techniques d'assistance médicale à la procréation (AMP), l'intérêt de la varicocélectomie dans de tels cas a été mis en question par certains auteurs [6]. D'autres, en revanche, ont rapporté récemment des cas d'induction de la spermatogenèse ayant permis d'obtenir des grossesses spontanées ou avec AMP sans recourir à une extraction testiculaire de spermatozoïdes $[3,7]$.

Le but de ce travail était d'évaluer les résultats de la ligature haute rétropéritonéale des veines spermatiques chez les hommes infertiles ayant une varicocèle associée à une azoospermie non obstructive ou à une oligozoospermie sévère.

\section{Patients et méthodes}

De janvier 2005 à décembre 2007, 246 hommes ont subi une varicocélectomie au service d'urologie-andrologie du CHU Aristide-Le-Dantec de Dakar. Ces patients étaient pour la plupart envoyés au service par des gynécologues, des sages-femmes ou des médecins généralistes. Ils ont été examinés en s'intéressant entre autres à la position, à la consistance et au volume des testicules. Les cordons spermatiques ont été palpés en station debout et lors de la manœuvre de Valsalva. Le diagnostic de la varicocèle a été posé sur la base des résultats de l'examen physique et de l'échographiedoppler des bourses. Cette varicocèle a été classée en trois grades selon la graduation de Dubin et Amelar [8]. Dans les formes bilatérales, cette graduation avait tenu compte du côté le plus dilaté. L'oligozoospermie a été définie comme une concentration de spermatozoïdes inférieure à 5 millions/ml [3]. L'azoospermie a été confirmée par deux spermogrammes à au moins 70 jours d'intervalle. Un bilan hormonal a été effectué chez les patients ayant une azoospermie. Les valeurs normales étaient comprises entre 2 et $10 \mathrm{mUI} / \mathrm{ml}$ pour la follicle-stimulating hormone (FSH) et entre 2,8 et $8 \mathrm{ng} / \mathrm{ml}$ pour la testostéronémie totale.

Dans le cadre de cette étude rétrospective, nous avons retenu les dossiers des patients ayant réuni les quatre critères suivants :

- une infertilité de couple définie comme étant une impossibilité d'obtenir une grossesse après au moins 12 mois de rapports sexuels réguliers, non protégés ;

- une épouse âgée de moins de 45 ans sans troubles menstruels et dont le bilan d'infertilité (examen physique, hormonologie, hystérosalpingographie) est normal ;

- une varicocèle clinique sur un côté au moins. Les patients qui avaient une varicocèle clinique gauche et infraclinique droite ont été inclus et classés parmi les varicocèles bilatérales ;

- des spermogrammes pré- et postopératoires réalisés dans un des deux laboratoires suivants : le laboratoire de l'institut Pasteur de Dakar et le laboratoire de cytologie, cytogénétique et biologie de la reproduction de la faculté de médecine de Dakar. Le recueil et l'examen du sperme dans ces laboratoires sont effectués selon les recommandations de l'OMS [9].

Nous avons ainsi colligé 61 patients dont 46 (75,4\%) présentaient une oligozoospermie sévère (groupe I) et 15 (24,6\%) une azoospermie non obstructive (groupe II). L'infertilité était primaire dans 49 cas et secondaire dans 
12 cas. Ces patients avaient tous eu une ligature haute rétropéritonéale sous anesthésie locorégionale des veines spermatiques selon la technique de Palomo [10] avec préservation des artères spermatiques.

Les patients ayant une éventuelle azoospermie obstructive ont été exclus de cette étude en se basant sur les antécédents du patient (orchiépididymite chronique, prostatite chronique, traumatismes des bourses), sur les résultats de l'examen physique (palpation des canaux déférents et épididymes) et sur les résultats des examens complémentaires (volume de l'éjaculat, taux de FSH).

Après l'intervention chirurgicale, les résultats de la varicocélectomie ont été appréciés sur la base des spermogrammes postopératoires. Dans le groupe I, ces spermogrammes ont été réalisés quatre mois (22 cas), huit mois (19 cas) et 12 mois (cinq cas) après l'intervention. Parmi les patients de ce groupe I, quatre avaient fait deux spermogrammes postopératoires, et un patient en avait effectué trois. Chez ces patients, nous avons tenu compte du spermogramme dans lequel la concentration des spermatozoïdes était la plus élevée. Dans le groupe II, les patients n'avaient effectué qu'un spermogramme postopératoire quatre mois (neuf cas) et huit mois (six cas) après la varicocélectomie.

L'analyse statistique a été effectuée à l'aide du logiciel ÉpiInfo ${ }^{\mathrm{TM}}$ version 2000.

\section{Résultats}

Les données cliniques des patients sont rapportées au Tableau 1. Dans le groupe I et en préopératoire, la concentration moyenne des spermatozoïdes était de 1,85 $\pm 1,40$ millions/ml. La moyenne des pourcentages de spermatozoïdes de mobilité normale était de 43,3 $\pm 21,5 \%$.

Tableau 1 Données cliniques des patients

\begin{tabular}{|lll|}
\hline Aspects cliniques & $\begin{array}{l}\text { Oligozoospermie } \\
\text { sévère } \\
(\boldsymbol{n}=\mathbf{4 6})\end{array}$ & $\begin{array}{l}\text { Azoospermie } \\
\text { non obstructive } \\
(\boldsymbol{n}=\mathbf{1 5})\end{array}$ \\
\hline Âge moyen des & $35,5 \pm 6,4$ & $40,8 \pm 7,2$ \\
patients (ans) & $(23-47)$ & $(27-47)$ \\
Âge moyen des & $27,4 \pm 6,03$ & $30 \pm 6,3$ \\
épouses (ans) & $(19-38)$ & $(20-39)$ \\
Durée moyenne & $4,9 \pm 3,4$ & $6 \pm 3,1$ \\
de l'infertilité (ans) & $(1-13)$ & $(3-15)$ \\
Site de la varicocèle $(\%)$ & & \\
Bilatérale & 89,1 & 86,7 \\
Unilatérale gauche & 10,9 & 13,3 \\
Grade de la varicocèle $(\%)$ & & 20 \\
Grade I & 4,3 & 66,7 \\
Grade II & 84,7 & 13,3 \\
Grade III & 10,9 & \\
\hline
\end{tabular}

Les spermatozoïdes de morphologie anormale représentaient $65,05 \pm 21,6 \%$ en moyenne. Après varicocélectomie, la concentration moyenne des spermatozoïdes était de $8,3 \pm 10,3$ millions $/ \mathrm{ml}$. La mobilité normale était en moyenne de 47,6 $\pm 29,2 \%$, et les spermatozoïdes de morphologie anormale représentaient $50,08 \pm 26,9 \%$ (Fig. 1). Chez un patient (concentration préopératoire de spermatozoïdes : 1 million/ml), le spermogramme postopératoire à six mois avait trouvé une azoospermie. Après un suivi postopératoire moyen de 26,2 \pm 11,6 mois, 12 couples $(26,1 \%)$ avaient rapporté des grossesses spontanées. Parmi ces couples, 11 avaient une infertilité primaire et un avait une infertilité secondaire.

Dans le groupe II, en préopératoire, le taux de FSH moyen était de 19,1 $\pm 19,1 \mathrm{mUI} / \mathrm{ml}(5,6-71,08 \mathrm{mUI} / \mathrm{ml})$, et la testostéronémie totale était en moyenne de $5,5 \pm 3,5 \mathrm{ng} / \mathrm{ml}$ (1-11,2 ng/ml). Après varicocélectomie, une induction de la spermatogenèse a été observée chez trois patients $(20 \%)$. Les concentrations de spermatozoïdes (spermogrammes postopératoires à quatre mois) chez ces trois patients étaient de 2,1 millions/ml, $0,1 \mathrm{million} / \mathrm{ml}$ et $0,01 \mathrm{million} / \mathrm{ml}$. Les pourcentages de spermatozoïdes mobiles étaient respectivement de 35, 20 et $25 \%$. Parmi ces trois patients, seul le couple du second groupe avait rapporté une grossesse spontanée (dix mois après varicocélectomie) qui s'est terminée par une fausse couche.

Sur l'ensemble des 61 patients de cette série, des complications postopératoires ont été observées dans cinq cas $(8,2 \%)$. Il s'agissait de deux cas de varicocèle résiduelle ou récidivée, de deux cas d'hydrocèle et d'un cas d'infection de la plaie opératoire.

\section{Discussion}

La varicocèle est une affection fréquente chez les hommes infertiles. Elle a été identifiée chez 35 \% des hommes ayant

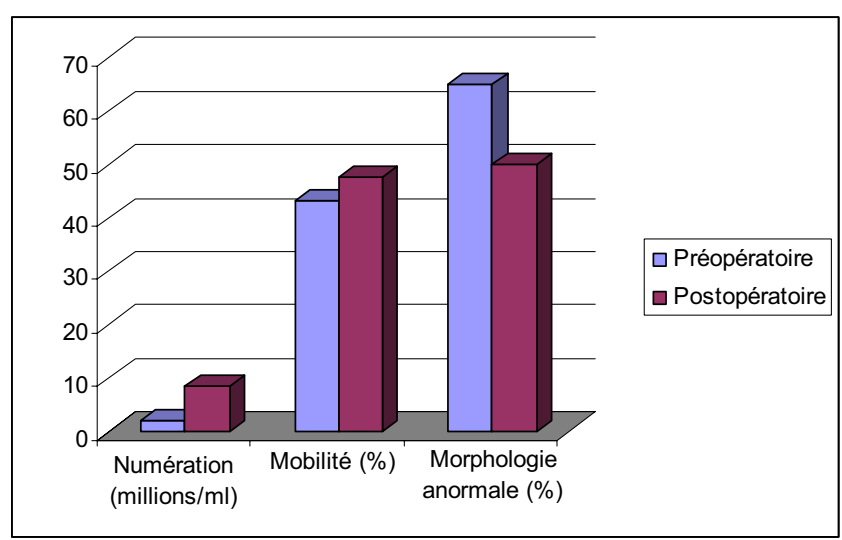

Fig. 1 Évolution des paramètres spermatiques dans le groupe des 46 patients ayant une varicocèle associée à une oligozoospermie sévère 
une infertilité primaire et chez $81 \%$ des hommes ayant une infertilité secondaire [2]. De janvier 2005 à décembre 2007, 246 hommes avaient subi une varicocélectomie au service d'urologie-andrologie du CHU Aristide-Le-Dantec de Dakar. Seuls $24,7 \%$ de ces patients ont été recrutés dans le cadre de cette étude. Les autres ne correspondaient pas aux critères d'inclusion. Il s'agissait pour l'essentiel de patients ayant une numération de spermatozoïdes supérieure à 5 millions $/ \mathrm{ml}$ ou de varicocélectomies pour douleurs scrotales chez des patients célibataires. D'autres patients ont été exclus parce qu'il existait un facteur féminin d'infertilité du couple ou parce qu'aucune donnée sur l'épouse n'était disponible. Dans notre contexte, du fait des conséquences sociales de l'infertilité masculine, beaucoup de patients consultent souvent seuls, parfois à l'insu de leurs épouses.

L'existence d'une relation causale entre varicocèle et infertilité masculine fait toujours l'objet d'un débat. Mordel et al. [11] dans une revue de 50 études portant sur 5471 hommes infertiles avaient trouvé $57 \%$ d'améliorations des paramètres du spermogramme après varicocélectomie et $36 \%$ de grossesses spontanées chez leurs partenaires. Mais aucune de ces 50 études n'a été contrôlée, et un taux de grossesses spontanées de $33 \%$ a été rapporté dans une revue incluant 2026 couples hypofertiles, non explorés, non traités [12]. Dans une autre revue, Schlesinger et al. [13] avaient rapporté un taux de grossesses variant entre 0 et $70 \%$ après varicocélectomie toutes techniques confondues.

Sur les 61 patients inclus, $54(88,5 \%)$ avaient une varicocèle bilatérale. Un résultat comparable a été trouvé par plusieurs auteurs [7,14]. Gat et al. [15] recommandent de considérer la varicocèle comme une pathologie bilatérale et d'explorer attentivement les hommes ayant une varicocèle clinique gauche à la recherche d'une atteinte bilatérale. Dans notre étude (les deux groupes réunis), la varicocèle était de grade I dans cinq cas $(8,1 \%)$, de grade II dans 49 cas $(80,3 \%)$ et de grade III dans sept cas $(11,4 \%)$. La corrélation entre le grade de la varicocèle et l'ampleur des améliorations du spermogramme après varicocélectomie n'a pas été formellement établie. Certains auteurs ont trouvé que plus la dilatation de la varicocèle est grande, plus les améliorations du spermogramme après traitement sont importantes [16]. En revanche, d'autres auteurs n'ont pas trouvé de différence significative des améliorations du spermogramme en fonction du grade de la varicocèle [17].

Les améliorations du spermogramme observées ici sont comparables à celles rapportées par de nombreux auteurs $[3,7]$. Mais la comparaison des études doit tenir compte de l'absence de définition consensuelle de l'oligozoospermie sévère [18]. Matthews et al. [7], par exemple, l'ont définie comme une concentration de spermatozoïdes inférieure à $1 \mathrm{million} / \mathrm{ml}$. Les améliorations du spermogramme rappor- tées par Gat et al. [14] sont nettement plus élevées que celles de notre étude. Cela peut s'expliquer par trois raisons : le long délai de consultation chez nos patients (6 $\pm 3,1$ ans dans le groupe azoospermie), la réalisation parfois tardive des spermogrammes postopératoires et d'éventuelles anomalies génétiques chez nos patients. En effet, chez certains patients du groupe II, le spermogramme de contrôle a été fait huit mois après la chirurgie ; or, il a été rapporté qu'en cas d'induction de la spermatogenèse, les hommes ayant une azoospermie non obstructive pouvaient redevenir azoospermiques six mois après la varicocélectomie [14,19], d'où l'intérêt de l'autoconservation des spermatozoïdes. Nous avons trouvé un taux de grossesses spontanées de $26,1 \%$ dans le groupe oligozoospermie et une seule grossesse dans le groupe azoospermie. Ces taux sont comparables à ceux rapportés dans d'autres études $[3,7]$ bien que la technique chirurgicale utilisée dans ces études soit différente de la nôtre. En effet, Matthews et al. avaient rapporté un taux de grossesses spontanées de $19 \%$ chez 22 hommes ayant une azoospermie et 56 ayant une oligozoospermie sévère [7].

Selon Cayan et al. [20], une induction de la spermatogenèse n'est possible chez les hommes ayant une azoospermie non obstructive que s'ils n'ont pas d'anomalies génétiques associées. Mais nous n'avions pas réalisé d'explorations génétiques (caryotype, microdélétions sur le bras long du chromosome Y [Yq]) chez nos patients à cause des coûts élevés de ces explorations et de l'absence de prise en charge par une assurance maladie. Ces explorations étaient pourtant nécessaires, car l'oligozoospermie sévère et l'azoospermie non obstructive sont des déficits quantitatifs de la spermatogenèse qui doivent amener à suspecter la présence d'une anomalie génétique sous-jacente $[21,22]$. Les microdélétions du chromosome Y sont fréquentes chez les patients dont l'infertilité est idiopathique, mais elles peuvent aussi être associées à d'autres causes d'infertilité comme la varicocèle, la cryptorchidie ou l'orchite [23]. La présence de ces facteurs étiologiques n'exclut donc pas de rechercher ces microdélétions. Pour certains auteurs, leur présence peut être considérée comme la cause de l'infertilité et permet aux cliniciens et aux patients d'éviter des bilans et des traitements médicaux ou chirurgicaux inutiles [24]. Les techniques d'AMP ne sont pas encore disponibles dans notre pays, raison pour laquelle aucun de nos patients n'en a fait recours. Cette situation est regrettable dans la mesure où ces techniques ont permis ailleurs d'augmenter considérablement le taux de grossesses après varicocélectomie chez des hommes ayant une azoospermie non obstructive $[7,14]$.

L'impossibilité dans notre contexte d'identifier les patients porteurs d'anomalies génétiques et de recourir aux techniques d'AMP constitue les principales limites de cette étude en plus du fait qu'elle soit rétrospective, sans groupe témoin comme la plupart des études sur la varicocèle. 


\section{Conclusion}

La varicocélectomie chez les hommes infertiles ayant une azoospermie non obstructive entraine parfois une induction de la spermatogenèse avec apparition dans l'éjaculat de spermatozoïdes mobiles utilisables dans le cadre d'une AMP. Elle permet donc dans certains cas d'éviter le recours à l'extraction testiculaire des spermatozoïdes. Chez les hommes ayant une oligozoospermie sévère, la varicocélectomie améliore parfois les paramètres du spermogramme et la fertilité. La réalisation de cette intervention chez de tels hommes est donc une option raisonnable. Cette étude pose par ailleurs le problème de la disponibilité de la biologie moléculaire et des techniques d'AMP dans les pays sous-développés.

Conflit d'intérêt : aucun.

\section{Références}

1. Gonzales R, Reddy P, Kaye KW, Narayan P (1983) Comparison of Doppler examination and retrograde spermatic venography in the diagnosis of varicocele. Fertil Steril 40:96-9

2. Gorelick JL, Goldstein M (1993) Loss of fertility in men with varicocele. Fertil Steril 59:613-6

3. Ishikawa T, Kondo Y, Yamaguchi K, et al (2008) Effect of varicocelectomy on patients with unobstructive azoospermia and oligospermia. BJU Int 101:216-8

4. Crosignani PG, Rubin BL (2000) Optimal use of infertility diagnostic tests and treatments. The ESHRE Capri Workshop Group. Hum Reprod 15:723-32

5. Tulloch WS (1953) Consideration of sterility, subfertility in the male. Edinb Med J 59:29-34

6. Schlegel PN, Kaufman J (2004) Role of varicocelectomy in nonobstructive azoospermia. Fertil Steril 81:1585-8

7. Matthews GJ, Matthews ED, Goldstein M (1998) Induction of spermatogenesis and achievement of pregnancy after microsurgical varicocelectomy in men with azoospermia and severe oligoasthenospermia. Fertil Steril 70:71-5
8. Dubin L, Amelar RD (1971) Etiologic factors in 1294 consecutive cases of male infertility. Fertil Steril 22:469-74

9. World Health Organisation (1999) WHO Laboratory manual for the examination of human semen and sperm-cervical mucus interaction 4th ed. Cambridge University Press, Cambridge UK

10. Palomo A (1949) Radical cure of varicocele by a new technique: preliminary report. J Urol 61(3):604-7

11. Mordel N, Mor-Yosef S, Margalioth EJ, et al (1990) Spermatic vein ligation as treatment for male infertility. J Reprod Med 35:123-7

12. Taylor PJ, Collins JA (1992) Unexplained infertility. Oxford University Press, Oxford

13. Schlesinger MH, Wilets IF, Nagler HM (1994) Treatment outcome after varicocelectomy. A critical analysis. Urol Clin North Am 21:517-29

14. Gat Y, Bachar GN, Everaert K, et al (2005) Induction of spermatogenesis in men after internal spermatic vein embolization for the treatment of varicocele. Hum Reprod 20:1013-7

15. Gat Y, Bachar GN, Zukerman Z, et al (2004) Varicocele: a bilateral disease. Fertil Steril 81:424-9

16. Steckel J, Dicker AP, Goldstein M (1993) Relationship between varicocele size and response to varicocelectomy. J Urol 149:769-71

17. Jungwirth A, Gögüs C, Hauser W, et al (2001) Clinical outcome of microsurgical subinguinal varicocelectomy in infertile men. Andrologia 33:71-4

18. Grimes DA, Lopez LM (2007) "Oligozoospermia" "azoospermia" and other semen-analysis terminology: the need for better science. Fertil Steril 88:1491-4

19. Pasqualotto FF, Sobreiro BP, Hallak J, et al (2006) Induction of spermatogenesis in azoospermic men after varicocelectomy repair: an update. Fertil Steril 85:635-9

20. Cayan S, Lee D, Black LD, et al (2001) Response to varicocelectomy in oligospermic men with and without defined genetic infertility. Urology 57:530-5

21. Bourrouillou G, Bujan L, Calvas P, et al (1992) Rôle et contribution du caryotype dans l'infertilité masculine. Prog Urol 2:189-95

22. Wallerand H, Bernardini S, Chabannes E, Bittard H (2003) Infertilité masculine de cause génétique et biologie moléculaire. Prog Urol 13:560-3

23. Krausz C, Quintana-murci L, Barbaux S, et al (1999) A high frequency of $\mathrm{Y}$ chromosome deletions in males with nonidiopathic infertility. J Clin Endocrinol Metab 84:3606-12

24. McElreavey K, Krausz C, Patrat C, Fellous M (2002) Infertilité masculine et microdélétions du chromosome Y. Gynecol Obstet Fertil 30(5):405-12 